\title{
Guía de viajes alrededor de la alcoba de Vicente Quirarte
}

\section{Travel guide around the bedroom of Vicente Quirarte}

\author{
Ignacio Ballester Pardo ${ }^{1}$
}

Como citar este artículo: Ballester Pardo, I. (2021). Guía de viajes alrededor de la alcoba de Vicente Quirarte, Pangeas. Revista Interdisciplinar de Ecocrítica (núm. 3) 26-36. https://doi.org/10.14198/PANGEAS.18847

\begin{abstract}
Resumen
Vicente Quirarte (Ciudad de México, 1954) reunió algunos de sus ensayos sobre viajes, viajeros y caminos literarios en Viajes alrededor de la alcoba (1993). Este libro toma por título la máxima de otro poeta mexicano, Xavier Villaurrutia. Desarrolla la idea clásica de quien se mueve desde su habitación con la lectura de crónicas que le llevan a imaginar un mundo más saludable. A partir de ciertos motivos itinerantes que recorre Vicente Quirarte -como el flâneur de Charles Baudelaire o Walter Benjamin, el doppelgänger de Arthur Rimbaud, el homeless de Leopold Bloom o Die Verwandlung de Franz Kafka- estableceremos una poética de la ecocrítica urbana que se viene defendiendo en la lírica mexicana. La reescritura genera un diálogo constante entre diversos tiempos y espacios. Atendemos a distintas perspectivas del viaje: como proceso de escritura, desde la historia o la arquitectura, el viaje físico o conjetural, hasta llegar a la infancia como separación, iniciación y retorno. Si nos fijamos en la tradición que hereda y renueva el poeta, advertimos un elogio del espacio urbano (Ciudad de México, Praga o Nueva York) y de los animales (el perro, la ballena o las hormigas) en contra de ese final voluntario que es el suicidio. Se trata de elementos también presentes en Alejandro Tarrab o Esther M. García. La literatura permite establecer un viaje interior que beneficie a la vida en comunidad, con una conciencia del medio, solo entre la multitud.
\end{abstract}

Palabras clave: Itinerancia; estática; poesía; México; ecocrítica.

\begin{abstract}
Vicente Quirarte (Mexico City, 1954) collected some of his essays about travel, travelers and literary paths in Viajes alrededor de la alcoba (1993). The title of this book is due to another Mexican poet, Xavier Villaurrutia. It develops the classic idea of the author who travels in his room by reading chronicles that lead him to imagine a healthier world. Starting from certain itinerant motifs that Vicente Quirarte travels -like the flâneur of Charles Baudelaire or Walter Benjamin, the doppelgänger of Arthur Rimbaud, the homeless of Leopold Bloom or Die Verwandlung of Franz Kafkawe will establish a poetic of urban ecocriticism that has been defending itself in the Mexican lyric. This rewriting generates a constant dialogue between different times and spaces. Diverse perspectives of the trip are observed: as a writing process, from history or architecture, the physical or conjectural journey, until we reach childhood, separation, initiation and return. If the tradition that the poet inherits and renews is looked at, we notice an appraisal of urban space (Mexico City, Prague or New York) and of some animals (the dog, the whale or the ants) against the voluntary end, which is suicide. These elements are also present in Alejandro Tarrab or Esther M. García's works. Literature allows us to establish an interior journey that benefits life in community, being aware of the environment, only among the crowd.
\end{abstract}

Key words: Itinerant; static; poetry; Mexico; ecocriticism.

1. Ignacio Ballester Pardo. Universidad de Alicante. ignacio.ballester@ua.es https://orcid.org/0000-0002-5826-3167 
El viaje se ha limitado en los últimos meses a la imaginación. Durante la pandemia, la literatura - esto es, la ficción de la mano del relato, la memoria y el deseo- como desplazamiento y atención al medio que nos rodea, a favor de la habitabilidad, se ha visto reducida a la letra impresa y, cada vez más, al contacto virtual que podemos rastrear desde el país con más hispanohablantes.

\section{INTRODUCCIÓN}

Vicente Quirarte (Ciudad de México, 1954) publica en 1993 su libro de ensayos Viajes alrededor de la alcoba. Toma el título del también poeta mexicano Xavier Villaurrutia, cuyo nombre tiene el máximo galardón que dos años antes, en 1991, recibió — junto a Gerardo Denizpor su poemario El ángel es vampiro. A través del caso particular del escritor y académico desglosaremos mediante imágenes (verbales), cual guía de caminantes, algunas etapas que explican la poética del viaje en México desde y hasta distintas tradiciones.

Los diez textos del título que Quirarte toma de Villaurrutia - después de que este hiciera lo propio con Paul Morand ("La tête au Pole, les pieds sur l'Equateur, quoi qu'on fasse, c'est toujours le voyage autor de ma chambre", 9) y antes, aunque no lo recoge aquí, también con Xavier de Maistre en 1794- desglosan con claridad el itinerario de quien se dedica a la literatura desde diversos géneros. Por este motivo, aludiremos a su poesía (ahora ya no) completa en Razones del samurai (2000). Haremos lo propio también con el ensayo (también considerado novela o memorias) que le dedica a su padre, el historiador Martín Quirarte, titulado La Invencible (2012), donde reconoce lo siguente:

A punto de vencerme el sueño, uno de mis juegos infantiles consistía en imaginar mi cama como una barca por el cielo que al mismo tiempo no abandonaba la habitación en que dormía. Entonces no sabía de la existencia de un poeta llamado Xavier Villaurrutia ni de su concepto aprendido en Paul Morand y antes en Xavier de Meistre [sic] — de que los emprendemos hacia el interior de nosotros y que todo viaje se realiza primero alrededor de la alcoba ([2012b] 2015: 97).

La búsqueda "hacia el interior de nosotros" cristalizará en una preocupación por lo externo; comprometiéndose, después de una travesía mental, con el medio, el contexto más cercano. En buena parte de su obra el viaje aparece desde distintas perspectivas que iremos analizando también a partir de la novela sobre los viajeros de México a EE UU en el siglo XIX, La isla tiene forma de ballena (2015), o la obra de teatro Melville en Mazatlán (2015).

Cada una de las tres secciones de Viajes alrededor de la alcoba -o cada andén, como los llama Quirarte en el prólogo- hace alusión a la estructura de la prolífica obra que crea desde hace más de cuarenta años; nos referimos a los rites de passage que toma de Arnold van Gennep: separación, iniciación y retorno (2000: 282-283). Como vemos, para el mexicano la poética necesariamente parte de la tradición y continúa en el texto, en el viaje. Defiende y elogia, así, el ambiente que nos precede y nos rodea, en un círculo infinito: con la definición de ecocrítica que da Benito Elías García-Valero a Laura Díaz (2021): "relación entre literatura y medio ambiente; y la cuestión del lenguaje. Los seres humanos mediante el lenguaje construimos una serie de manifestaciones de lo que es el mundo natural" (4:25). Es decir: "la ecocrítica sería el estudio de los textos que se han producido donde la naturaleza aparece representada para ver de qué manera hemos concebido la naturaleza a lo largo del tiempo" (5:40). Seguiremos dicha representación en la obra de Quirarte, la cual de manera inductiva puede ofrecer un panorama de lo que sucede en el siglo XXI con la literatura mexicana.

\section{GUÍA DE VIAJES}

\subsection{El viaje como proceso de escritura}

Para Quirarte, el viaje comienza con la decisión de ponerse a escribir, de armar un texto como hoja de ruta o bitácora. La alegoría conecta directamente 
con su poética: escribir es siempre, al menos, un traslado; a veces de regreso. Es en los primeros años, junto a sus hermanos y en contacto directo con el centro de la Ciudad de México, cuando el poeta viaja con la lectura y siente la necesidad de escribir, recordar, construir, atravesar, crear y alimentar estos otros mundos:

\begin{abstract}
Mis hermanos y yo no nos perdíamos un solo número de una historieta titulada Grandes viajes, donde aprendimos los nombres y las hazañas de Marco Polo, Roald Amundsen y Charles Lindbergh. Entre nuestras películas predilectas se hallaban Veinte mil leguas de viaje submarino, Viaje al centro de la tierra y 2001: Una odisea espacial. Por el centro de la Ciudad de México emprendíamos exhaustivas caminatas en involuntario homenaje al cuento "De los Apeninos a los Andes" incluido en Corazón de Edmundo de Amicis. Lectura igualmente inolvidable fue El maravilloso viaje de Nils Holgersson a través de Suecia de Selma Lagerlöf. Aunque los viajes más lejanos que emprendíamos eran a Guadalajara, una vez a bordo del pullman imaginábamos que el tren sería asaltado por Francisco Villa o Lawrence de Arabia, o que en el interior viviríamos la intriga de una historia de Agatha Christie o de Sherlock Holmes (1993: 13).
\end{abstract}

Esta memoria que Quirarte incluirá en el capítulo VII de La Invencible son las primeras líneas que aparecieron en Viajes alrededor de la alcoba. Asimismo, la continuación de tal testamento literario que publica en México en 2012 y en España en 2015 (seguimos esta última edición) recoge parte del prólogo de la obra de 1993, que tomamos como referencia para este trabajo. Y es que dicho libro, que publicó el Gobierno del Estado de Nuevo León, Monterrey, aparentemente periférico, es fundamental para el desarrollo poético de un autor que a finales del siglo XX se doctoró en la Universidad Nacional Autónoma de México con su tesis Elogio de la calle. Biografía literaria de la Ciudad de México. 1850-1992 (2001): el cuidado de una vida urbana a través de la historia literaria.

La poesía que reúne en Razones del samurai (2000: 9-13) comienza con los quince puntos de su poética, también presentes en el primer capítulo de Viajes alrededor de la alcoba (1993: 19-23), ambos titulados "Urgencia de la poesía" (disponible en la red) y con mínimas variaciones que son citas a demás poetas que complementan, casi veinte años después, un sentir insustituible; por ejemplo, en la segunda máxima:

II. La poesía es el tren de los ausentes. Sin horario fijo, invade los andenes o aparece, imprevista, en mitad del desierto. Arranca nuestras raíces para volvernos parte de su vértigo, en escasas ocasiones nos obliga a viajar entre sus ruedas. A cambio nos concede la alegría y la libertad heroica de los vagos (1993: 19).

Y añade esta cita en su poesía reunida: "Confiesa Eduardo Hurtado: "Aquí estoy. Tengo mi oficio./ Jefe de la estación,/ sin silbato y sin horario fijo,con corridas continuas al pavor del desierto" (2000: 9). De este modo, pese a ser iluminadores los versos del también poeta capitalino de los cincuenta, Quirarte, a finales del siglo pasado, ya tiene clara la definición de lo poético; una acepción que, ligada al viaje, parte de un nutrido y variado compendio de lecturas (mexicanas, españolas, francesas o inglesas) y se irá desarrollando en sus diversos géneros literarios. Los puntos XIV y XVI de "Urgencia de la poesía", sobre el amor y la felicidad de quien se dedica a la escritura, se suman a los quince de la edición de Viajes alrededor de la alcoba.

Doce serán las características del proceso de escritura, en general, que desglosa en La Invencible a partir precisamente del pionero en los viajes alrededor de la alcoba, Xavier de Maistre, y del primer poeta moderno en México, López Velarde, que "caminaba por las calles de la ciudad nocturna en busca de la palabra justa donde podía acomodarse y encontrar permanencia la emoción efímera" (2015: 51). La cercanía que empiezan a tener con las universidades quienes nacen en la segunda mitad del siglo xx permite un acceso a lecturas que irán formando su poética, pues: "10. Leer es otra forma de escribir. Es volver a escribir. No repetir la intención del autor, sino hallar para el texto los caminos que el escritor acaso ni siquiera concibió" (2015: 53). Tales caminos imprevistos conectan la ciudad con movimientos complejos (Lynch, [1960] 1998: 146), hasta oníricos.

La poética que estudiamos de Quirarte (Ballester, 2015), la seguimos ahora desde la perspectiva ecocrítica, como viaje y cuidado desde el lenguaje verbal en la historia literaria. 
El mexicano dialoga con sus referentes, entre quienes se encuentra Emily Dickinson. La intertextualidad se logra con las numerosas citas que fusionan el ensayo y la narrativa en el poema. A la estadounidense (protagonista de un reciente poemario de Jorge Esquinca, de 2015: Cámara nupcial ) pertenece el acápite de "Ética y estética del amador de libros”, el segundo capítulo de Viajes alrededor de la alcoba:

No hay, como el libro, una

fragata para llevarnos lejos,

no hay transporte comparable

a una página de furiosa poesía.

Semejante trayecto puede hacerlo

el más pobre sin oprimir su bolsa;

qué frugal el carruaje que a un

alma humana lleva (25).

Y es que la escritura es el viaje de salvación de lo íntimo a lo social: "Escribir un libro es un acto solitario. Aunque su autor se encuentre acompañado por sus antecesores escriturarios o por sus íntimos fantasmas, se halla más solo que el náufrago perdido en el Atlántico" (1993: 25). Generalmente, la literatura es un tránsito de quien escribe o lee solo entre la multitud, como el flâneur de Charles Baudelaire o Walter Benjamin, el doppelgänger de Arthur Rimbaud, el homeless de Leopold Bloom o Die Verwandlung de Franz Kafka. La transformación del texto y con el texto nos llevará de la mano de Quirarte a México, Praga o Nueva York, desdoblándose en animales (destacando la ballena, el perro o las hormigas) que abogan por el cuidado del planeta en todas sus formas. La misma poética puede extraerse del acto de lectura, solitario, recluido en la alcoba.

La Odisea de Homero, El Quijote de Cervantes, el Ulysses de Joyce, el flâneur de Baudelaire o Benjamin, así como el desdoblamiento de Rimbaud, la transformación de Kafka, el laberinto de Borges o la palinodia de Fernando del Paso, van enriqueciendo el viaje de quien se encuentra desde el espacio urbano con poetas más recientes:

Escribir es semejante a esta comunión de los sentidos con la ciudad que descubrimos y hacemos poco a poco nuestra. La lectura como escritura es un viaje de la imaginación cultivada, y aunque leamos en la fila del banco, formulemos esquemas mientras entrenamos para resistir un maratón o descifremos la oscuridad de un enigma en medio del combate amoroso, las palabras y acciones de los otros terminan por fijarse en el estatismo aparente de la alcoba. Escribir es viajar y viajar es leer y traducir. Lo sentí, sin saberlo, al enfrentar, también en mi infancia, por vez primera un ensayo y no descifrar ninguno de sus misterios. Así como hay gente que no merece viajar, el mal servidor de las palabras no se enterará de que ha viajado. Los neoplatónicos leyeron La Odisea como una gran alegoría del alma. A partir de esa exigencia es preciso emprender todo traslado: tan definitivo puede resultar a un hombre ir a comprar el periódico, como a otro tan fútil recorrer el mundo. Las veinticuatro horas de Leopold Bloom en el Ulysses de Joyce nos enseñan más que los cien años pasivos de quien elige la cobardía del sedentario sobre la imprudencia del vagabundo. Leerse en las diversas realidades del mundo y traducirlas a un lenguaje objetivo, es como la reconstrucción del viaje. Y si los viajes, como los amores, son ingratos, el emprendido en nombre de la imaginación y la curiosidad es el único que recompensa desde el andén. Gracias a la imaginación nos es concedido viajar sin movernos de nuestro sitio y conocer más lugares que los permitidos por el desplazamiento en el espacio. Sólo el lector enamorado consigue boletos para el vagón de primera de ese tren ([2012b] 2015: 98-99).

El dominio de otras lenguas como el inglés o el francés le permite al mexicano incorporar a la alegoría del viaje como escritura el concepto de la traducción, pues el trasvase de un texto (de un espacio) a otra lengua (otro código) arranca con la creación de una realidad paralela al origen del que se parte; es decir: un desplazamiento. Si se puede viajar desde la lectura, sin despegarse de un libro, para la escritura es necesario moverse y emprender un viaje que nos permita reconocer este planeta y dar con aquello que queramos plasmar y mejorar con la poesía, la vida. Quien lee parece "dispuesto a enfrentar la casi imposible hazaña de viajar sin moverse de su sitio, de crear mundos más habitables que éste" (2015: 49). Con tal utopía la realidad parece otra. Habitar y 
amar el espacio recorrido es un propósito literario a través de los tiempos, las tradiciones literarias que en Quirarte ligan la escritura con el viaje.

\subsection{El viaje necesita la historia}

A propósito del anterior complemento directo que podría ser sujeto, y viceversa, en 2013, en su casa de la Ciudad de México, en una entrevista (Ballester, 2016), le preguntamos al poeta si la historia tenía o debía tener influencia en la literatura; a lo que respondió aludiendo al primer capítulo de su libro de relatos Un paraguas y una máquina de coser, titulado "Su majestad la Historia" (2010: 15-20) por el historiador francès Jules Michelet, estudioso de "Sa Majesté, L'Histoire". Este texto ya aparecía con mínimas modificaciones (marcades entre corchetes) en el ensayo "La historia como madre de la imaginación" (1993: 39-48) y hace hincapié en viajes, viajeros y caminos:

\footnotetext{
En el invierno de 1965 Visión panorámica de la Historia de México de Martín Quirarte vio su primera edición. La alegría personal de mi padre y la colectiva de la familia se vio para mí ensombrecida por la circunstancia de que, a causa de las vacaciones, mi padre ["el flamante autor" (2010: 15)] me impuso la tarea de resumir diariamente uno de los capítulos de su libro ["que integraban a su nuevo hijo" (2010: 15)]. De tal modo, mi primera disciplina escritural no fue la Literatura sino la Historia. A la prosa exacta y exigente de Martín Quirarte, a su poderío sintético debo, entonces, mi inicial gozo de la Historia y la convicción de que, al ser abordada con pasión e inteligencia, es más apasionante que todas las ficciones. Prefiero las Vidas paralelas de Plutarco a las Vidas imaginarias de Marcel Schwob. Me emociona más el realismo de las Revistas históricas sobre la Intervención francesa en México de José María Iglesias que las fantasías de Vicente Riva Palacio y Juan Antonio Mateos (1993: 39-40).
}

En los libros de viajes escritos por mexicanos (abordados por el propio autor en el reciente ciclo "Poética de la crónica. De la Ciudad de los Palacios a la Ciudad de los Batracios" que impartió en la UNAM) destacan otros trabajos del poeta que estudiamos: Jerusalén a la vista. Tres viajeros mexicanos a Tierra Santa (2003) - Más allá de la visión de Anáhuac. Poética de los viajeros mexicanos (2007) y Republicanos en otro imperio. Viajeros mexicanos a Nueva York 1830-1895 (Ballester, 2019). Este último ensayo histórico daría pie a la novela, también histórica, La isla tiene forma de ballena. A pesar del surrealismo que tanto atrae a Quirarte y permea en jóvenes poetas de México, quien crea nuevos caminos literarios, vitales o ecocríticos debe de conocer el pasado para no repetir y errar el presente; de ahí que la base de lo fantástico sea siempre real. Cuando El Hombre Araña pasea por la ciudad, vemos a un periodista por la calle insomne de Nueva York; del mismo modo que una ballena en Mazatlán (pensamos en Herman Melville) permite el diálogo y hasta el debate entre un sujeto poético que se desdobla en el tan característico poema de máscara para la reciente tradición mexicana (Higashi, 2015: 354-355). El desdoblamiento, a través de distintas voces narrativas o la apropiación en el sujeto poético, supone a la vez un desplazamiento, en el tiempo $y$ en el espacio; a favor de la habitabilidad, precisamente, por el devenir de la literatura tradicionalmente política que ahora definimos como cívica.

\subsection{El viaje y su arquitectura}

La interdisciplinariedad resulta uno de los rumbos que viene aprovechando en las últimas décadas la poesía mexicana. Nuevamente, Vicente Quirarte hace lo propio desde la historia de su padre, Martín Quirarte. De él heredó la sintaxis que sostiene un relato fantástico por su rigor. En este sentido, la imaginación nos permite reivindicar las virtudes de una ciudad estoica, la Ciudad de México, que cada día supera las adversidades, como cuenta el poeta en La ciudad como cuerpo (1999): otro símil de las arterias que recorren quienes viajan a pequeña escala movidos por la pasión. Uno de tales textos se titula "Arquitectura hechizada (7:00 A. M.)" y fragmentariamente dice: "Amenazada pero invencible, la vieja ciudad es nutrida por las resurrecciones de sus habitantes permanentes o sus amantes de paso" (1999b: 26). Citadina o turística, la sociedad construye un imaginario a favor de la vida urbana en una de las regiones 
más masificadas y contaminadas. A tal propósito dedicó Quirarte el Festival de "Mextrópoli" de EI Colegio Nacional en 2017.

No obstante, la lírica mexicana apuesta con fuerza por el ámbito rural, por la vida en la naturaleza cuando se impone una reclusión que vencen en Campeche poetas como Briceida Cuevas Cob; o, en Hidalgo, Rosa Maqueda Vicente. El texto se entiende como cobijo que opera a favor de un viaje (así lo sintió Quirarte al escribir Fra Filippo Lippi: Cancionero de Lucrezia Buti, pues motivó su viaje a Italia en los ochenta) o el viaje mental tras este, cual saudade, al trazar hipotéticos días del autor de Moby Dick o Bartleby, el escribiente en Jerusalén (2020).

\subsection{El viaje físico}

Ligado con la arquitectura, el viaje físico conecta el cuerpo (humano, animal o urbano) con los desplazamientos (interiores, instintivos o geográficos). Vicente Quirarte describe este proceso literario y ecocrítico, una vez más, en Viajes alrededor de la alcoba y en La Invencible. Aunque seguimos la edición más reciente de La Invencible, incluimos partes que no se han conservado de Viajes alrededor de la alcoba:

Viví mis primeros quince años en relación estrecha con piedras y fantasmas de la vieja ciudad. Cuando daba mis primeros pasos en ella, Agustín Yáñez escribía Ojerosa y pintada. En la novela, el conductor no actúa, en sentido estricto, pero su oído registra las voces de la urbe. Artistas, limosneros, prostitutas, obreros desfilan en el aparador rodante del taxi para conformar un mural sonoro de los años cincuenta. ["Dos décadas más tarde, Luis Arturo Ramos escribirá Violeta-Perú, nombre de una línea de autobuses urbanos, donde sus personajes, [sic] marginados a quienes torturan monstruos internos y exteriores, miran la ciudad desde el autobús. Al igual que el "Ómnibus" de Julio Cortázar, en Luis Arturo Ramos el medio colectivo de transporte se convierte en espacio iniciático, vehículo del viaje interior realizado por los pasajeros (1993: 154-155)] Cuando nací, hacía 10 años que Efraín Huerta había publicado Los hombres del alba, primer libro de poemas íntegramente dedicado a la ciudad de México. De esa época es también el cuadro panorámico de Juan O'Gorman donde aparece la capital vista desde el monumento a la Revolución. Es la urbe que ha rebasado su traza tradicional, para incorporarse vertiginosamente a la economía de posguerra. Es una ciudad sonriente y plácida, provinciana no obstante sus conquistas, y bajo cuya fachada se desatan historias que sólo pueden generarse en el universo de la ciudad. Es la ciudad donde Cantinflas estrena Gran Hotel, pero también aquella donde los niños perdidos de Huerta llevan "en vez de corazón, un perro enloquecido." Es la ciudad aún a escala humana, pero ya con los principios de gigantismo que Ilevará a Salvador Novo a exclamar: "uno llega, si vive en [la ciudad] muchos años, a no ejercer más que unos cuantos sitios" ([2012b] 2015: 90).

Las distintas escalas de la ciudad nos hacen imaginar y ver a Vicente Quirarte escribiendo en el espacio físico de su poema-novela-ensayo. La Invencible se la dedicó a su padre, el historiador Martín Quirarte, después de que en 1980 se tirara de un puente en Ciudad Universitaria. Más de treinta años después, reconstruye esos caminos finales que conectan distintas urbes en una misma capital y lo hace desde la cantina homónima (La Invencible) que frecuentaba, en la colonia San Ángel, al sur de la ciudad, donde el poeta es peatón comprometido con el medio ambiente. Quirarte no es propiamente un flâneur. Su propósito no es perderse en la ciudad, sino someterla, caminarla y recorrerla hasta dominarla. Es una actualización del paseante parisino en la ciudad de México. "El poeta no es sólo un paseante ocasional, sino un consciente e incansable trotamundos" (Bravo Varela, 2008: 32). Le ocurre lo mismo que a Rimbaud, cuya finalidad -como demuestra Marco Antonio Campos- "no es causar asombro. Lo que verdaderamente le interesa (¿interesar es un verbo aplicable a Rimbaud?) no es el uso del espacio, sino su conquista" (Quirarte, 2005: 27). Y aquí el autor se refiere al sujeto lírico de sus poemas. El genio francés prefería el trayecto al destino: "le gustaban más los caminos que las ciudades, los senderos y no la llegada" (108); se inclinaba por el "aquí y ahora" (141) al final del poema "Tarde bajo el kiosko”, que Quirarte dedicar al precoz francés "con una indagación en las raíces psíquicas del 
sujeto poético, que transita desde la imagen sesgada del paseante o flâneur baudeleriano hasta la reivindicación de una identidad urbana como huella cívica" (Bagué, 2006: 222). Dicha "huella cívica" establecerá en México unas pautas de ciudadanía o de comportamiento en comunidad que desde las referencias culturales de diversos países establecerán con el viaje una defensa de la habitabilidad urbana (pero también rural). El peatón o peatonauta (Castillo, 2014) sería el correlato del flâneur y del dandi en Quirarte: "este andariego poeta que, desde siempre, intuyó a la urbe como un ente orgánico" (Piña Zentella, 2008: 26).

El viaje por la ciudad se organiza en las tres fases de Arnold van Gennep: separación, iniciación y retorno. La estructura de Quirarte es común en otras poéticas recientes. El hecho de que El nombre de esta casa (1999) de Julián Herbert se divida en tres partes ("Gentes", "Calles" y "Letreros") recuerda a la estructura tripartita de las prosas que reunió Quirarte en Enseres para sobrevivir en la ciudad: "Enseres", "Para sobrevivir" y "En la ciudad". En este camino, el también poeta peatón Omar Pimienta escribe una crónica cotidiana en "La libertad es una zona afectada", de La libertad: ciudad de paso (2006): "Camino por estas calles: / alguien atropelló el semáforo nuevo; / hay negocios de Internet donde antes fondas; / mataron a Roberto por andar de narco; / mandan saludar a mi padre" (7). El problema de la frontera provoca una evolución urbana que registra el poema. Según veíamos con Julián Herbert a raíz de Quirarte, la división tripartita permite el viaje por el espacio urbano carente de civismo, en el caso de Pimienta advertimos una división tripartita similar: "La Libertad", "De Paso" y "Ciudad"; separación, iniciación y retorno.

El cuerpo que permite un viaje por sus correspondientes articulaciones dialoga con otras poéticas: Datsun (2009), de Xitlalitl Rodríguez Mendoza, Tránsito (2011), de Claudina Domingo, Babia (2011), de Karen Villeda, o Atardecer en los suburbios (2011), de Minerva Reynosa; estudiadas con tino por Manuel de J. Jiménez en "Tránsitos y cruces (viajes de la nueva poesía mexicana)" (2012). En tales obras las coordenadas espacio-temporales rigen poemarios que cartografían y discurren por la ciudad (real o imaginada) y defienden una moral cívica (posible o imposible) en contacto, por ejemplo, con animales o infraestructuras que desde el estado sólido plantean nuevas posibilidades para la poética y la vida del siglo XXI.

\subsection{El viaje conjetural}

Con el "superhistoriador" de Ramón Gómez de la Serna, el poeta mezcla lo que sucedió con lo que pudo ocurrir. José Emilio Pacheco denomina a los poemas conjeturales -Jorge Luis Borges dixit- como ucronía o "historias de la vida irreal" (Quirarte, 2010: 59). El interés de este último por la historia lo animó a imaginar el ya mencionado Fra Filippo Lippi: Cancionero de Lucrezia Buti (1982). De tal modo lo reconoce en Viajes alrededor de la alcoba (1993):

Mi primer intento consistió en reconstruir
con base en algunos datos reales, la relación
amorosa entre el pintor renacentista Filippo
Lippi y su modelo, la monja Lucrezia Buti. A lo
largo de 20 sonetos y un poema extenso, más
cercano a la reflexión que al canto, quise dar
testimonio del inicio, la evolución y la cima de
esa historia individual, inserta ya para siempre
en la Historia, como puede verlo cualquiera en la
galería de los Uffizzi o en la millonaria industria
que ha reproducido la Madonna en todos los
formatos. Del mismo modo he explorado la
historia conjetural de personajes como Marco
Polo, Miguel Angel, Ramón López Velarde, Jean-
Arthur Rimbaudo Julio Torri (47).

Arturo Trejo dialoga con el autor a propósito de los entresijos del poemario: "El Cancionero de Lucrezia Buti es un libro hecho antes de conocer Italia. En 1977, junto con el poeta Héctor Carreto, nos metimos al Museo de las Culturas y, en la sección de artes plásticas, nos sentamos frente a la madona de Filippo Lippi” (1982b: 19). En ese momento surgió el interés por hacer la historia conjetural de un pintor que se enamora de la modelo, tal como le ocurría al joven poeta en su vida real. Dicha historia ya se advierte al final del libro anterior, Calle nuestra (1979), con el poema "Posdata para Fra Filippo Lippi" (2000: 104), dedicado precisamente a Héctor Carreto. Así pues, una galería en México inspira el poemario y el posterior viaje a Italia. Los siguientes son los 
últimos versos Fra Filippo Lippi: Cancionero de Lucrezia Buti:

Por eso no olvido, mi madona, por eso no pidas, mi Lucrezia, que olvide el viaje por ese océano [abierto, márcame para siempre con tus ojos y después de la herida nazca el [mundo (1982: 109).

El museo vuelve a ser el escenario para dar alas a la imaginación y unir a dos personajes básicos para la historia y la formación literaria de Quirarte: Marx y Rimbaud. El alemán y el francés se pudieron haber encontrado en las escaleras del British Museum, como testimonia el diario de Jenny, la hija de Marx. Quirarte recoge la traducción que su "amigo Javier García-Galiano" (1993: 52) hace de este documento y reflexiona al respecto. El civismo aboga por una defensa ecocrítica del viaje vertical, entre generaciones:

\footnotetext{
En los tiempos de Marx y Rimbaud, Londres era la ciudad más grande del mundo, aquella que ilustraba, mejor que ninguna otra, los esplendores y miserias de la moderna vida urbana. Rimbaud emprende una radiografía de la ciudad recorriéndola en todas sus direcciones. Podemos establecer con cierta precisión su bitácora de viaje gracias a las cartas de Verlaine, donde da cuenta de la variedad de tipos, lugares y rincones que junto con su joven amigo descubre (1993: 58).
}

En este sentido, la obra de teatro Melville en Mazatlán (2015) o la novela La isla tiene forma de ballena (2015) conjeturan la presencia del autor de Moby Dick en tierras mexicanas (como hará con el poemario Melville en Jerusalem en 2020), de la misma manera que vemos a Marx y a Rimbaud cruzarse en Londres. Ambos hechos son verosímiles porque parten de un hecho histórico y lo sitúan en un espacio físico real, fácilmente ubicable por quien lee la historia, el relato o el poema.

\subsection{El viaje a la infancia}

La orfandad de Luis Cernuda en México, la oscuridad de César Vallejo en Francia, el compromiso de Pablo Neruda en España o la necesidad literaria de María
Zambrano desde la pluralidad del sujeto poético conforman las alas con las que Vicente Quirarte emprende este vuelo, cual Vicente Huidobro en Altazor (Quirarte, 1993: 116). Volver a nacer es la lectura de cada viaje en sus diversos géneros literarios:

Con todo, el narrador urbano enfrenta una exigencia suprema: dejar de ser un name dropper para volver autónoma la ciudad que aparece en el cuerpo de su narración: la ciudad como entidad viva y actuante y no como escenografía. De ahí la vida que tiene el Dublín recorrido por Leopold Bloom; se trata de una ciudad viva, tanto por el personaje como por nosotros, sus acompañantes. El Ulises moderno no vive en la ciudad; vive la ciudad: es la ciudad. Ese "Man of the crowd”, cuyo antecedente ilustre está en el cuento de Poe, evoluciona hacia el "Wakefield" de Nathaniel Hawthorne y logra su madurez en el "Bartleby" de Herman Melville, es un habitante relativamente nuevo en la historia de la literatura. Es el usuario de la ciudad, que encuentra en la caminata su heroísmo (1993: 164).

El superhombre camina la ciudad, que es distinto a caminar en ella o porella (Quirarte, [1994] 2012a: 36; [2012b] 2015: 58). Las referencias de Viajes alrededor de la alcoba dibujan un mapa de las constelaciones que orientan a Quirarte y a la poesía mexicana contemporánea. Esther M. García (Ciudad Juárez, Chihuahua, 1987) en una prosa yuxtapuesta se pone en la piel del feto que se pierde en la frontera entre México y EE UU: "Realidad ensangrentada hecha violencia hecha jirones una infancia rota He visto la luz roja que me guiará. Soy yo el que decide quien vive o muere. Soy Barrabás pisando la arena ardiente mientras degüello a un hombre. Soy Judas Iscariote besando la sangre de la cruz" (García, [2013] 2014a: 34); mientras que Alejandro Tarrab (Ciudad de México, 1972) recupera el recuerdo que tiene de niño, cuando su abuela se suicidó: "Hay viajeros que al llegar a un lugar no sólo se postran (genu flectere) para besar la tierra, cogen un puñado del suelo y lo tragan. Esto propicia una unión; la identificación con el espacio insólito que intentan habitar. Lo mismo cuando se parte" (Tarrab, 2015: 48). Es más: "Ciertos suicidas se arrodillan para morder algo de este polvo y lo que comienza ahí, en la [sic] entrañas, es un hormigueo: animales 
ardientes caminando al interior del cuerpo. Un adelanto de lo que vendrá más tarde: el regreso, el sabor del barro cocido en la entraña ardiente de la madre" (Tarrab, 2015: 48). A partir de las pautas que Quirarte ofrece para el viaje y la poesía, encontramos poetas que sostienen sus recientes obras a través de la memoria y crean un testimonio que visibiliza la violencia contra la maternidad o la reflexión en torno a la muerte voluntaria.

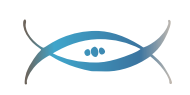

\section{CONCLUSIONES}

Desde Cervantes, sor Juana Inés de la Cruz, Xavier de Maistre, Virginia Woolf, Xavier Villaurrutia o Gabriel Fernández Ledesma (Pitman: 2008), viajar alrededor de la alcoba -desde la habitación que se queda sin velas al iPad que se nos escurre de los dedos durante el confinamiento, es decir: el viaje de la lectura- ha ido despertando un tópico que explica desde la lírica, desde Quirarte, el proceso de escritura, la historia, la arquitectura, la física, la conjetura y la infancia, como necesidades y puntos de encuentro fundamentales para construir un mundo más habitable también con perspectiva ecocrítica. Desde el sueño, la razón no produce monstruos, sino que permite acercar lo lejano o, al menos, imaginarlo. Contra la melancolía, la interdisciplinariedad ofrece caminos aparentemente ficticios al callejón sin salida. La habitación no es únicamente el dormitorio. Más que la cama, urgen una mesa, una biblioteca, un museo. Si Quirarte escribe Fra Filippo Lippi: Cancionero de Lucrezia Buti sin estar en Italia, su viaje le permite conocer finalmente el Mediterráneo y plantear desde el amor un bálsamo -a la manera de Gilberto Owencontra el suicidio - que sufrirá por su padre- 0 la violencia de un estado de conciencia colectiva que continuará en poetas más recientes como Alejandro Tarrab o Esther M. García. El transcurso del poema bebe de la filosofía de Heráclito, pues todo cambia; y, al mismo tiempo, del eterno retorno de Friedrich Nietzsche y el uróboros. La separación, el inicio y el retorno de todo viaje ofrecen, para el poeta mexicano, un posible cambio en contra, por ejemplo, del suicidio de su padre y elegir así otro camino, trazado también por demás poetas de México como el reciente libro de Fernando Fernández, Viaje alrededor de mi escritorio (2020), donde reúne una década de trabajo en su blog Siglo en la brisa. La ecocrítica, al cabo, se basa en lo tangible, pero también debe de prestar atención a las abstracciones mentales con las que la sociedad del siglo xx। contamina nuestra convivencia solos entre la multitud.

En dicho sentido, a propósito de los más recientes estudios de poesía mexicana contemporánea, destaca la labor de Carmen Alemany Bay al frente del proyecto CORPYCEM de la Universidad de Alicante. Su investigación en torno a la poeta ñähñu Rosa Maqueda Vicente y la ecocrítica fue presentada en el Congreso "Looking for Experiences and Practices for an Ecological Civilization in Latin America and the Caribbean", que en 2021 organizó el Instituto de estudios Latinoamericanos de HUFS, de la Universidad coreana Kyung Hee.

De esta manera, mediante la literatura el autor o la autora establece un reducto que salvaguarda las referencias y las artes necesarias para el ánimo y la vida. Asimismo, quien lee tiene a su alcance, en el texto, una guía no solo para imaginar que viaja y se mueve y conoce lugares lejanos en otra época, sino que crece y alimenta la defensa de lo único que nos une durante el aislamiento, la memoria. Perderla ha sido uno de los temores de quienes escriben sus crónicas de viajes, desde que se fijó la escritura cuneiforme en Mesopotamia tras las civilizaciones nómadas. Dar cuenta de ella, de la realidad, a través de la ficción, de la literariedad que convierte un suceso histórico o nimio en novela o poema permite que continuemos moviéndonos, desplazándonos, acercándonos.

\section{BIBLIOGRAFÍA}

BALLESTER PARDO, I. (2015). “Arte poética en Vicente Quirarte: decálogo entre el cielo y la tierra", en Carmen Alemany Bay (coord.), Artes poéticas mexicanas (de los Contemporáneos a la actualidad). Guadalajara: Universidad de Guadalajara, 265-281.

BALLESTER PARDO, I. (2016). "Entrevista a Vicente Quirarte: al centro del margen", en Cuadernos de Aleph. 8, 185-192. 
BALLESTER PARDO, I. (2019). La dimensión cívica en la poesía mexicana contemporánea: herencia, tradición y renovación en la obra de Vicente Quirarte. Tirant lo Blanch / Universidad Autónoma del Estado de México.

BAUDELAIRE, C. (2001). Las flores del mal, trad. I. Caparrós). Granada: Alhulia.

BAGUÉ QUÍLEZ, L. (2006). Poesía en pie de paz. Modos del compromiso hacia el tercer milenio. Valencia: Pre-Textos.

BENJAMIN, W. ([1969] 1990). Imaginación y sociedad. Iluminaciones I, trad. J. Aguirre. Madrid: Taurus.

BRAVO VARELA, H. (2008). Lo orillados. México: DGE Equilibrista / UNAM / Conaculta.

CASTILLO, R. (2014). "Brevísima y veloz presentación del peatonauta", en Casa del tiempo. 8, 31-33. Consultado en línea (27/02/2016): http://www.uam.mx/ difusion/casadeltiempo/08 sep_2014/ casa del tiempo eV num 8 31 33.pdf

DOMINGO, C. (2011). Tránsito. México: Fondo Editorial Tierra Adentro / Conaculta.

ESQUINCA, J. (2015). Cámara nupcial. México: Ediciones Era / Instituto Veracruzano de la Cultura.

FERNÁNDEZ LEDESMA, G. ([1938] 1992). Viaje Alrededor de mi Cuarto. Aguascalientes: Instituto Cultural de Aguascalientes / Gobierno del Estado de Aguascalientes.

GARCÍA, E. M. ([2013] 2014a). Sicarii. Saltillo: Instituto Municipal de Cultura de Saltillo. Consultado en línea (31/08/2018): https:// poesiamexa.wordpress.com/2016/04/02/ esther-m-garcia/

GARCÍA, E. M. (2014b). Bitácora de mujeres extrañas. México: Conaculta.

GARCÍA, E. M. (2017). Mamá es un animal negro que va de largo por las alcobas blancas. México: Universidad Autónoma del Estado de México. Consultado en línea (28/01/2018): http:// ri.uaemex.mx/handle/20.500.11799/67447

GUZMÁN, J. M.; LÓPEZ-PORTILLO Y ROJAS, J.; MALANCO, L. (2003). Jerusalén a la vista. Tres viajeros mexicanos a Tierra Santa, edición y prólogo de Vicente Quirarte. Toluca, Estado de México: Instituto Mexiquense de Cultura.

HERBERT, J. ([1999] 2013). El nombre de esta casa. México: Conaculta. Consultado en línea (15/02/2017): https://poesiamexa. wordpress.com/2016/04/27/julian-herbert/
HIGASHI, A. (2015). PM / XXI / 360. Crematística y estética de la poesía mexicana contemporánea en la era de la tradición de la ruptura. México: Universidad Autónoma Metropolitana / Tirant Lo Blanch.

DEJ.JIMÉNEZ, M. (2012). “Tránsitos y cruces (viajes de la nueva poesía mexicana)", en Crítica, 147. Consultado en línea (10/03/2017): http:// www.puntoenlinea.unam.mx/index.php/630

DÍAZ MACIÁ, L. (2012). "¿Qué es la ecocrítica? Entrevista a Benito García-Valero", en El viaje de Pequod. Consultado en línea (02/03/2021):https://www.ivoox.com/quees-ecocritica-audios-mp3_rf_66074386_1. html?fbclid=IwAR3jELO_iMN8KvjN9e6tW90 4dg7jivyZ2xwXi6BHLb-nIpvW6U1UwWeyR9M

JOYCE, J. ([1922] 2006). Ulises, trad. J. M. Valverde). Barcelona: Debolsillo.

KAFKA, F. ([1915] 2011). La metamorfosis, trad. E. San León Jiménez. Madrid: Diario Público.

LYNCH, K. ([1960] 1998). La imagen de la ciudad. Barcelona: Gustavo Gili.

PIMIENTA, O. (2006). La libertad: ciudad de paso. Tijuana: Centro Cultural Tijuana / Conaculta. Consultado en línea (16/02/2017): https:// poesiamexa.wordpress.com/2016/06/08/ omar-pimienta/

PIÑA ZENTELLA, M. (2008). "Trazo urbano en la obra de Vicente Quirarte", en Revista de Literatura Mexicana Contemporánea. México: The University of Texas at El Paso / Tecnológico de Monterrey, 39 (XIV), octubrediciembre, 23-30.

PITMAN, T. (2008). Mexican Travel Writing. Oxford: Lang.

QUIRARTE, V. (1979). Calle nuestra. México: Universidad Nacional Autónoma de México.

QUIRARTE, V. (1982a). Fra Filippo Lippi: Cancionero de Lucrezia Buti. México: Ediciones Fin de siglo.

QUIRARTE, V. (1982b). "La literatura debe estar al servicio de la vida y no al revés, indica Vicente Quirarte", por Arturo Trejo, en Unomásuno. 18 de julio, 19.

QUIRARTE, V. (1991). El ángel es vampiro. México: Ediciones Toledo.

QUIRARTE, V. (1993). Viajes alrededor de la alcoba. Monterrey: El mono gramático (Colecciones editoriales del Gobierno de Nuevo León).

QUIRARTE, V. ([1994] 2012a). Enseres para sobrevivir en la ciudad. Colombia: Luna Libros. QUIRARTE, V. (1999a). “Urgencia de la poesía”, 
en Artes poéticas. Consultado en línea (19/06/2018): http://artespoeticas. librodenotas.com/artes/807/urgencia-dela-poesia-1999

QUIRARTE, V. (1999b). La ciudad como cuerpo. México: Institución de Seguridad y Servicios Sociales de los Trabajadores del Estado.

QUIRARTE, V. (2000). Razones del samurai. Poesía reunida 1978-1999. México: Universidad Nacional Autónoma de México.

QUIRARTE, V. (2001). Elogio de la calle. Biografía literaria de la Ciudad de México. 1850-1992. México: Cal y Arena.

QUIRARTE, V. (2004). Nuevos viajes extraordinarios. Puebla: Colibrí.

QUIRARTE, V. (2005). Del monstruo considerado como una de las bellas artes. México: Paidós.

QUIRARTE, V. (2007). Más allá de la visión de Anáhuac: Poética de los viajeros mexicanos. Monterrey: Universidad Autónoma de Nuevo León.

QUIRARTE, V. (2009). Republicanos en otro imperio. Viajeros mexicanos a Nueva York 1830-1895. México: Universidad Nacional Autónoma de México.

QUIRARTE, V. (2010). Un paraguas y una máquina de coser. México: Terracota.

QUIRARTE, V. (2015a). La isla tiene forma de ballena. México: Seix Barral.

QUIRARTE, V. (2015b). Melville en Mazatlán. México: Ardiente Paciencia.

QUIRARTE, V. (2015c). La Invencible. México: Joaquín Mortiz, 2012b; Granada: Valparaíso Ediciones.

QUIRARTE, V. (2017a). Poética de la crónica: de la Ciudad de los Palacios a la Ciudad de Ios Batracios. México: Universidad Nacional Autónoma de México. Consultado en línea (16/06/2018): https://www.youtube.com/ watch?v=QnQs33RbkKE

QUIRARTE, V. (2017b). "Mesa de diálogo "EI arquitecto y la ciudad" (Mextrópoli)", en YouTube. México: El Colegio Nacional. Consultado en línea (16/06/2018): https:// www.youtube.com/watch?v=-V6169JkHkk

QUIRARTE, V. (2018). País Ilamado infancia. México: El Colegio Nacional. Consultadoen línea: https:// www.youtube.com/watch?v=sxLGuaK8jb4

REYNOSA, M. (2011). Atardecer en los suburbios. México: Fondo Editorial Tierra
Adentro / Conaculta. Consultado en línea (16/06/2018): https://poesiamexa.wordpress. com/2016/03/16/minerva-reynosa/

RIMBAUD, A. ([1988] 1955). Poesías y otros textos, trad. J. Abeleira. Madrid: Hiperión.

RODRÍGUEZ MENDOZA, X. (2009). Datsun. México: Punto de Partida, UNAM. Consultado en línea (16/06/2018): https://poesiamexa.wordpress. com/2016/04/18/xitlalitl-rodriguez-mendoza/

TARRAB, A. ([2006] 2009). Litane. México: Bonobos / Conaculta. Consultado en línea (24/02/2017): https://poesiamexa.wordpress. com/2016/03/19/alejandro-tarrab/

TARRAB, A. (2010). Degenerativa. México:Bonobos. Consultado en línea (24/02/2017): https:// poesiamexa.wordpress.com/2016/03/19/ alejandro-tarrab/

TARRAB, A. ([2015] 2017). Caída del búfalo sin nombre. Ensayo sobre el suicidio. México: Malpaís Ediciones, 2017. Consultado en línea: https:// poesiamexa.wordpress.com/2016/03/19/ alejandro-tarrab/

VILLAURRUTIA, X. (2006). Obra poética (Edición crítica y estudio introductorio de Rosa García Gutiérrez). Madrid: Hiperión.

VILLEDA, K. (2011). Babia. México: Punto de Partida, UNAM. 